\title{
Role Of The Delta Resonance In The Population Of Excited States In High-Energy Reactions
}

\author{
Zs. Podolyák* \\ University of Surrey \\ E-mail: z.podolyakesurrey.ac.uk
}

\section{C.M. Shand}

Department of Physics, University of Surrey, Guildford, GU2 7XH, UK

\section{N. Lalović}

Department of Physics, Lund University, S-22100 Lund, Sweden

GSI Helmholtzzentrum für Schwerionenforschung GmbH, D-64291 Darmstadt, Germany

\section{J. Gerl}

GSI Helmholtzzentrum für Schwerionenforschung GmbH, D-64291 Darmstadt, Germany

\section{Rudolph}

Department of Physics, Lund University, S-22100 Lund, Sweden

\section{and the PRESPEC-AGATA collaboration}

\begin{abstract}
The ${ }^{54} \mathrm{Fe}$ nucleus was populated from a ${ }^{56} \mathrm{Fe}$ beam with an energy of $E / A=500 \mathrm{MeV}$. The internal decay of the $10^{+}$metastable state via $\gamma$-ray emission was observed. The structure of this isomeric state has to involve at least four unpaired nucleons, therefore it cannot be populated in a simple two-neutron removal reaction from the ground state of ${ }^{56} \mathrm{Fe}$, and we suggest that it was populated via the decay of the $\Delta^{0}$ resonance into a proton. This process allows the population of fournucleon states, such as the observed isomer. Therefore, the population of the four-particle $10^{+}$ isomer in ${ }^{54} \mathrm{Fe}$ is a consequence of the quark structure of the nucleons. The possible use of this reaction mechanism for producing exotic nuclei is discussed.
\end{abstract}

The 26th International Nuclear Physics Conference

11-16 September, 2016

Adelaide, Australia

${ }^{*}$ Speaker. 


\section{Introduction}

Reactions between atomic nuclei are used to produce other nuclear species. Usually the constituent protons and neutrons are considered elementary particles, and consequently both proton and neutron numbers are conserved. However, neither protons nor neutrons are fundamental particles; they have a quark structure, and their own excited states. Their lowest energy excitation is the $\Delta$ resonance at $1232 \mathrm{MeV}$ [1], few hundreds of $\mathrm{MeV}$ higher than the groundstate-mass energy at $\sim 939 \mathrm{MeV}$. Therefore in collisions where the kinetic energy of a single nucleon is comparable or higher than this energy difference, the internal excitations of the nucleons can play a role.

Nuclear reaction in the energy regime of hundreds of $\mathrm{MeV}$ per nucleon are called fragmentation or spallation. It is one of the main processes used to produce previously unsynthetised nuclei $[2,3,4,5]$ (fission is the other one). Fragmentation is the process when a heavy nucleus impinging on a target nucleus loses nucleons and becomes a different nuclear specie. Considering nucleons as elementary particles, the fragmentation products will have fewer or equal number of protons and neutrons than the initial projectile. In other words, the reaction product is a fragment of the initial nucleus.

The first evidences that nuclear excitations play a role in reactions are from the 1980s. D. Bachelier and co-workers reported the "First observation of the $\Delta$ resonance in relativistic heavyion charge-exchange reactions" [6] in 1986. They observed that ${ }^{20} \mathrm{Ne}(\mathrm{Z}=\mathrm{N}=10)$ nuclei at an energy of $\mathrm{E} / \mathrm{A}=950 \mathrm{MeV}$ could be transformed into ${ }^{20} \mathrm{~F}(\mathrm{Z}=9, \mathrm{~N}=11)$ and into ${ }^{20} \mathrm{Na}(\mathrm{Z}=11, \mathrm{~N}=9)$. These reactions do not conserve proton and neutron numbers. The explanation is that when a proton is excited to the $\Delta^{+}$resonance, it can decay into a neutron emitting a positive pion; in effect the proton is turned into a neutron. This produces ${ }^{20} \mathrm{~F}$. Similarly the $\Delta^{0}$ resonance of the neutron can decay into a proton emitting a negative pion, which results in the production on ${ }^{20} \mathrm{Na}$. The emitted pion reduces the kinetic energy of the nucleus (the pion mass is $\sim 140 \mathrm{MeV}$ ), and indeed the nuclei produced in this way were measured to have an energy about $\sim 300 \mathrm{MeV}$ lower. Other higher lying nucleon excitations also can play a role. One should note that the same final reaction product can be also populated in so called quasi-elastic reactions, when a proton/neutron of the projectile is replaced by a neutron/proton from the target; however this mechanism does not alter the kinetic energy of the product. The role of nucleonic excitations was also observed in more recent experiments, e.g. ${ }_{83} \mathrm{Bi}$ isotopes [7] as well as $\mathrm{N}=127$ isotones [8] were produced form ${ }^{208} \mathrm{~Pb}_{126}$. The production of $\mathrm{N}=127$ isotones from lead illustrates how this process can be used to produce exotic neutron-rich nuclei. Presently, such charge-exchange reactions are under intense investigations, both experimentally [9] and theoretically [10].

Here we present results form an experiment where the population of an excited state is the consequence of the inner structure on the nucleons. The main findings of the study were published recently [11]. Here, additional details about the analysis are provided, together with an outlook.

\section{Experimental details and results}

The experiment was performed at GSI, Darmstadt, Germany. A primary beam of ${ }^{56} \mathrm{Fe}$ at an energy of $\mathrm{E} / \mathrm{A}=500 \mathrm{MeV}$ impinged on a thin, $662 \mathrm{mg} / \mathrm{cm}^{2}$, Be target. The reaction products were separated and identified with the Fragment Separator [12]. The separator was used in achromatic 
mode and optimised for the transmission of bare ${ }^{54} \mathrm{Fe}$ ions. The optical transmission was $100 \%$. The energy straggling in the thin target is small, therefore the momentum distribution of the reaction products is determined by the reaction mechanism. A total of 6.8 million ${ }^{54} \mathrm{Fe}$ ions were identified. These were slowed down and finally implanted into a plastic stopper. The stopper was surrounded by the AGATA gamma-ray detector array [13], operated in close configuration [14].

The delayed gamma-ray spectra associated with ${ }^{54} \mathrm{Fe}$, as well as the partial level scheme of this nucleus are shown in figure 1 . The 411,1330 and $1408 \mathrm{keV}$ transitions from the decay of the $10^{+}$isomeric state are clearly visible in the spectra. In addition, the spectra contain background peaks at $511 \mathrm{keV}, 1436 \mathrm{keV}$ and $1461 \mathrm{keV}$. The latter ones originate from the decay of ${ }^{138} \mathrm{La}$ (present in the $\mathrm{LaBr}_{3}$ detectors in the setup) and ${ }^{40} \mathrm{~K}$ (mainly form the walls). More intriguingly, there is a peak at $741 \mathrm{keV}$ marked with $*$ in the middle panel of figure 1 . This originates from the $I^{\pi}=3 / 2^{-} \mathrm{T}_{1 / 2}=63.5(14) \mathrm{ns}$ isomeric state, decaying into the $7 / 2^{-}$ground state, in ${ }^{53} \mathrm{Fe}$ [15]. It was populated from ${ }^{54} \mathrm{Fe}$ by removing one neutron during the implantation process (after the identification). Consequently, the intensity of the peak is proportional with the number of ${ }^{54} \mathrm{Fe}$ ions, and more intense in the middle of the distribution. We note, that the equivalent $3 / 2^{-}$isomeric state in the ${ }^{53} \mathrm{Co}$ mirror nucleus was identified in a similar way, produced during the stopping process by nucleon removal [16].
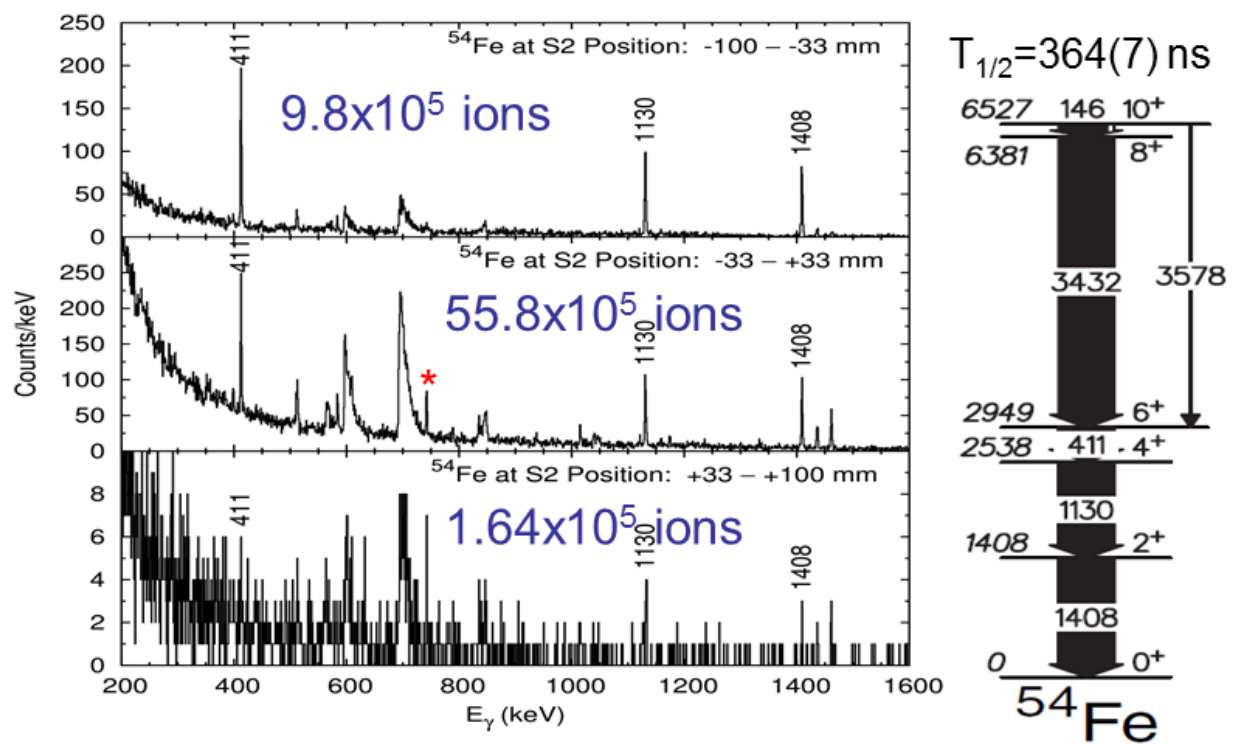

Figure 1: Delayed ( $\Delta t=117-1960 \mathrm{~ns}) \gamma$-ray spectra associated with ${ }^{54} \mathrm{Fe}$. The three panels correspond to ${ }^{54} \mathrm{Fe}$ ions with different parallel momentum ranges, as determined from the position at the S2 intermediate dispersive focal plane. (top) $\Delta p=-750,-247 \mathrm{MeV} / \mathrm{c} ;$ (middle) $\Delta p=-247,+247 \mathrm{MeV} / \mathrm{c} ;$ (bottom) $\Delta p=+247$, $+750 \mathrm{MeV} / \mathrm{c}$. The number of ${ }^{54} \mathrm{Fe}$ ions are indicated in each panel. The partial level scheme of ${ }^{54} \mathrm{Fe}$, showing the isomeric state and its decay is given on the right $[17,18]$.

In a pure fragmentation reaction, by removing two neutrons from the ground-state of ${ }^{56} \mathrm{Fe}$, only two-neutron states can be populated in ${ }^{54} \mathrm{Fe}$. Considering the available shell-model orbitals, the maximum spin of two-neutron states is generated by the $v f_{7 / 2}^{-2}$ configuration, and it is $I^{\pi}=6^{+}$. 
With two neutrons $I^{\pi}=10^{+}$can be obtained first with the $v h_{11 / 2}$ orbital, which is in the upper part of the $N=50-82$ shell. The $v h_{1}^{2} 1 / 2$ component of the $10^{+}$state can be estimated from the proton decay of the analogue $10^{+}$isomer in the mirror nucleus ${ }^{54} \mathrm{Ni}$. It is in the order of $10^{-6}[16]$. Therefore the $v h_{11 / 2}^{2}$ orbital is essentially empty; the configuration of the $10^{+}$isomer requires at least four particles [16]. And it cannot be populated by pure fragmentation of ${ }^{56} \mathrm{Fe}$. The mechanism of populating ${ }^{54} \mathrm{Fe}$ from ${ }^{56} \mathrm{Fe}$ at $\mathrm{E} / \mathrm{A}=500 \mathrm{MeV}$ is more complex.
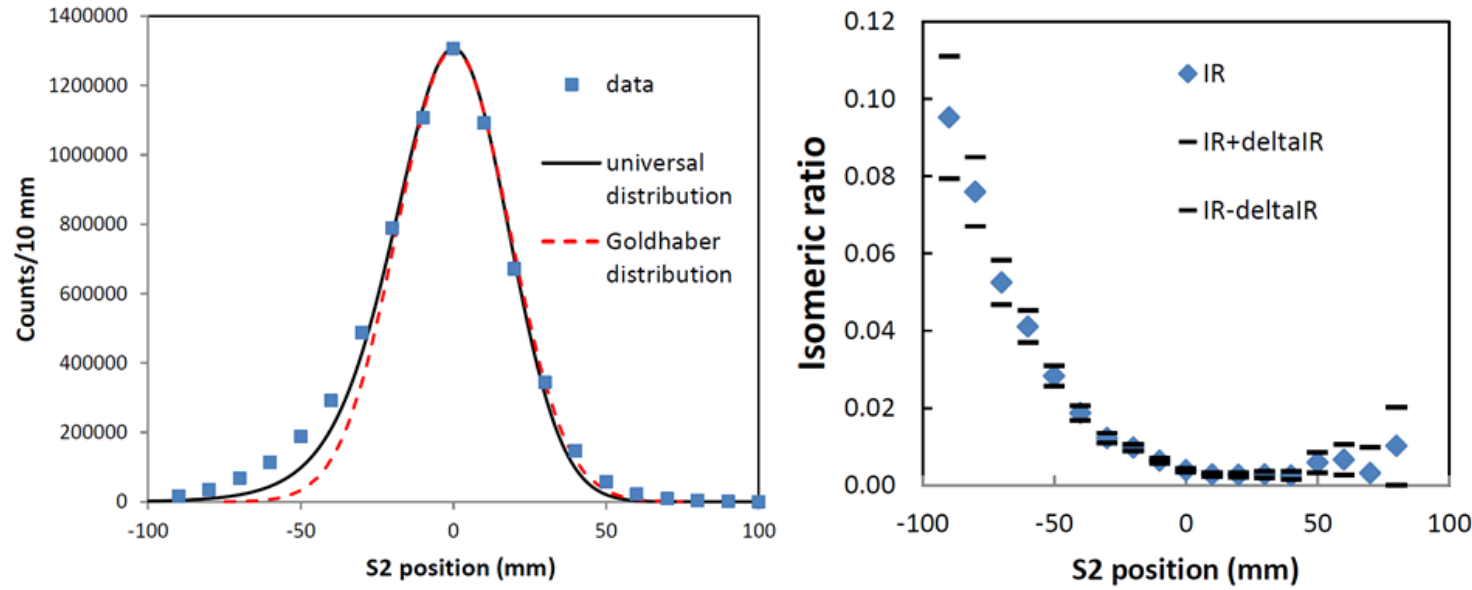

Figure 2: it (left): Position distribution of the ${ }^{54} \mathrm{Fe}$ ions at the intermediate focal plane of the fragment separator. The measured distribution is compared with the universal parametrisation of [19] (continuous line) and a symmetric distribution as given by the Goldhaber formula [20] (dashed line). (right): isomeric ratio as a function of position. The $\mathrm{S} 2$ position can be transformed into a parallel momentum distribution or kinetic energy loss distribution: $50 \mathrm{~mm}$ at $\mathrm{S} 2$ corresponds to $\Delta p=374.9 \mathrm{MeV} / \mathrm{c}, \Delta p / p_{0}=6.76 \times 10^{-3}$ and $\Delta E_{\text {kin }}=277.3 \mathrm{MeV}$.

From figure 1 it is clear that the isomer is produced mainly at negative horizontal positions at the intermediate S2 focal plane of the separator. This is a dispersive plane, therefore the position can be transformed into momentum. The distribution of the ${ }^{54} \mathrm{Fe}$ ions is shown on the left hand side of figure 2. This distribution is not symmetric, contrary to expectations. In such particle removal reactions at high energies the distribution is determined by the momentum of the removed nuclei, and therefore it should be symmetric [21]. The measured distribution has a small shoulder/tail at negative momentum shift. This tail is more obvious when the distribution is compared to the symmetric Goldhaber type distribution [20], also shown on the figure. The width of the Goldhaber distribution is determined by the used $\sigma_{0}=75 \mathrm{MeV}$ parameter. The distribution predicted by the so called universal distribution [19] is also shown. This is a parametrisation, with parameters deduced from experimental data. It predicts a very small low energy tail for our case of $\mathrm{E} / \mathrm{A}=500 \mathrm{MeV}$ bombarding energy, much smaller than the measured one.

The right hand side of figure 2 presents the determined isomeric ratio [22, 23] as function of position (momentum) at S2. It has relatively high values, in order of several percent at low momentum, but it is very small at positive momentum shift. This in contrast to what is expected for fragmentation reactions. In fragmentation the isomeric ratio is symmetric around $\Delta p=0$, with large values at high (negative and positive) momentum transfer, and minimum at no momentum transfer $[24,25]$. Clearly, the isomeric state is produced in a more complex way, not in the traditional 
fragmentation/nucleon removal reactions. To explain it we need to consider the internal excitations of nucleons.

The additional components to the fragmentation process can be disentangled considering that the fragmentation has close to symmetric parallel momentum distribution. The distribution of the non-fragmentation reaction as function of position is shown in figure 3. Here three different momentum distributions were considered for fragmentation: the symmetric Goldhaber distribution, the universal parametrisation and the right-hand side of the measured distribution. In all three cases the same picture arises, as seen in the three panels of figure 3. The large error bars are related to the uncertainty on where the middle of the measured distribution really is. An uncertainty of $1 \mathrm{~mm}$ was considered. By shifting the simulated distribution by $1 \mathrm{~mm}$, the number of nuclei changes a lot in the middle of the distribution, and therefore results in large error bars. In the tail of the distribution, $1 \mathrm{~mm}$ change makes a small change in the number of nuclei, and the uncertainty is therefore small. The non-fragmentation part represents $7-10 \%$ of the events. It peaks at position around -40 or -50 $\mathrm{mm}$, corresponding to a kinetic energy shift of $\sim 300 \mathrm{MeV}$.

Considering that only these additional processes can produce the isomer, the isomeric ratios are recalculated. These are also shown in figure 3. Again, the picture is similar in all three cases, with high isomeric ratio at high momentum transfer. At low (negative) momentum transfer the error bars are very large, so one cannot distinguish between a flat behaviour or a raising one.

We looked into other processes which can populate ${ }^{54} \mathrm{Fe}$. For example, two reaction processes, where in the first reaction ${ }^{55} \mathrm{Fe}$ is produced in the target, and in the second ${ }^{54} \mathrm{Fe}$ from ${ }^{55} \mathrm{Fe}$ either in the target or the degrader at S2. The yields were estimated. $0.5 \%$ of the events are from double reactions in the target. ${ }^{54} \mathrm{Fe}$ nuclei produced in the degrader from ${ }^{55} \mathrm{Fe}$ are much lower at around $0.03 \%$, as only a small fraction of ${ }^{55} \mathrm{Fe}$ makes it till the degrader. Importantly, these two-step processes would not populate the $10^{+}$state. In whatever way two neutrons are removed (in the same reaction or not), this state cannot be populated (only through the $h_{11 / 2}$ component discussed above) considering nucleons as elementary particles.

\section{Conclusions and outlook}

The experimental evidence, both the low momentum tail of the momentum distribution and the predominant production of the isomer in this tail corresponding to $\sim 300 \mathrm{MeV}$ energy shift, suggest that the $10^{+}$isomeric state is populated due to the internal excitation of the nucleus. In the high energy collision one of the protons of ${ }^{56} \mathrm{Fe}$ is excited into the $\Delta^{+}$resonance, which decays into a neutron emitting a positive pion. This process creates two unpaired particles. In addition two neutron are also removed from ${ }^{56} \mathrm{Fe}$ (either directly or evaporated), creating additional two unpaired nucleons. Consequently this process can populate four-particle states. We note that there is a weak production of the isomer also in the middle of the momentum distribution and at positive momentum transfer. These might be due to the aforementioned quasi-elastic processes and/or the $v h_{11 / 2}^{2}$ component of the isomeric state.

According to our knowledge, this is the first time that excited states produced as a result of the quark structure of the nucleons are studied. All previous experiments looked only at the final nucleus, and were not able to distinguish among different excited states. Here we were able to 

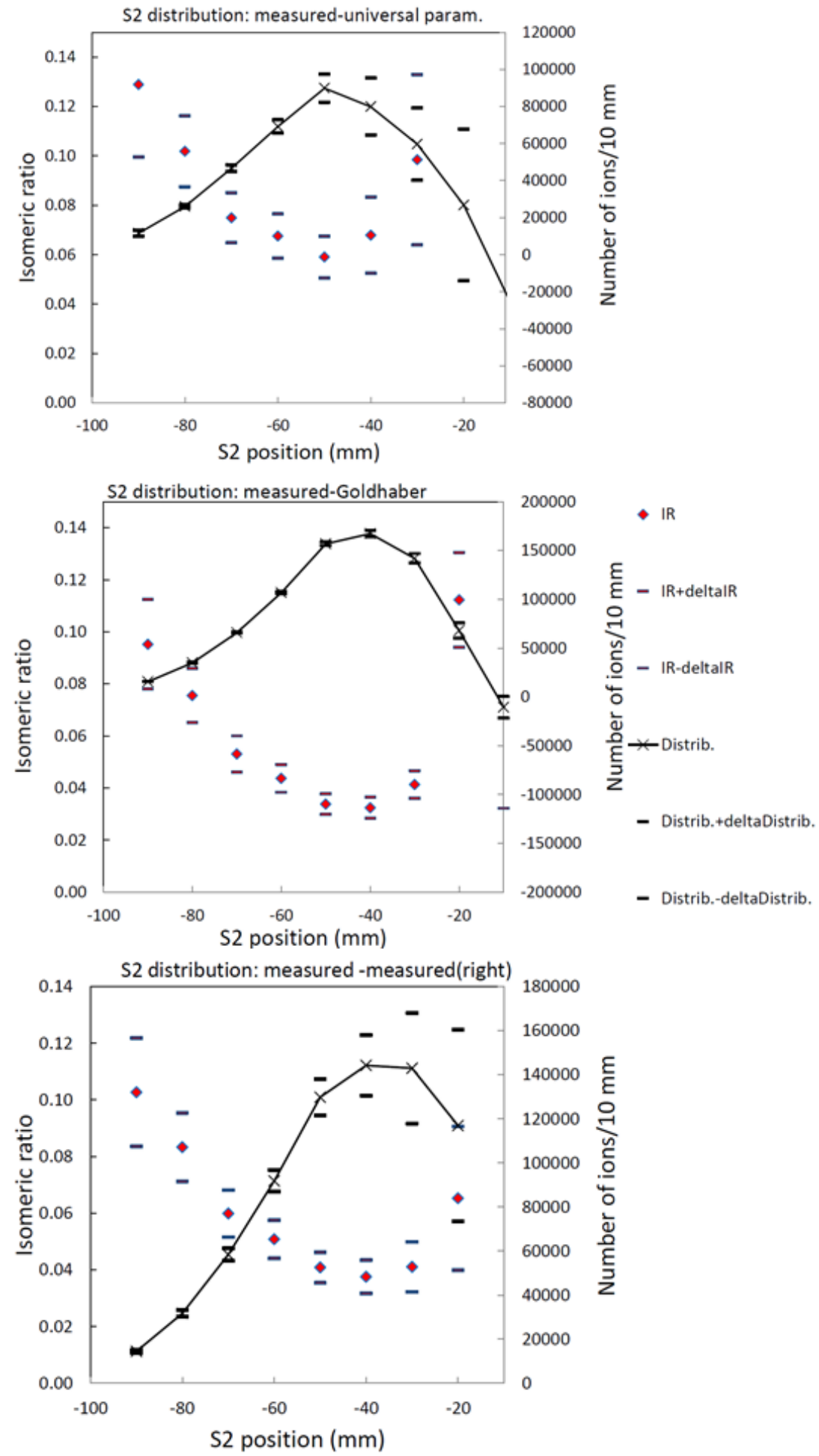

Figure 3: Isomeric ratio of the 'non-fragmentation' part of the reaction, after the pure fragmentation events are removed, as a function of position at the dispersive focal plane S2. The distribution of the nonfragmentation events is also shown. Three different methods were used to remove the fragmentation component (see text for details). The $-50 \mathrm{~mm} \mathrm{~S} 2$ position corresponds to $\Delta p=-374.9 \mathrm{MeV} / \mathrm{c}, \Delta p / p_{0}=-6.76 \times 10^{-3}$ and $\Delta E_{k i n}=-277.3 \mathrm{MeV}$. 
do it, thanks to the existence of a long-lived isomer in ${ }^{54} \mathrm{Fe}$, which allows the transport and the observation of its decay far from the production site.

As proved in the present study, excitation of the nucleon resonances increases the production of excited states with many unpaired nucleons. This will increase the isomeric ratio of high spin states. Experiments showed that high-spin states are populated with higher yield than expected from the theory of nuclear fragmentation. It was shown that the spin induced by the removed nucleons only, cannot account for the isomeric ratios of high-spin isomer states [26, 23, 27]. As shown here, excitations of the nucleonic resonances can produce additional spin. It is worth nothing that all experiments showing an excess of isomeric yield at high spin are from projectile fragmentation of ${ }^{238} \mathrm{U}$. The theoretical predictions depend strongly on the fission of the pre-fragments involved. A spin-dependent fissioning probability (where the spin has to be generated by the removed particles, not by rotation or aligned high $\mathrm{K}$ states), with reduced fission at high spin, could also be behind the discrepancy between experiment and theory.

The present study opens up the possibility to study the final nuclear states following the decay of nucleonic resonances in relativistic-energy heavy-ion collisions. The existence of a large number of isomeric states allows the extension of the present work to other regions of the nuclidic chart. Most promising are measurements focusing on nuclei with the same atomic mass as the projectile, as these allow the direct investigation of the process without the interference caused by additional nucleon emission. Finally we note that similar studies could be carried out by populating the nucleonic resonances via other processes, such as high energy photons.

Acknowledgements: We thank the GSI accelerator staff for their excellent work. This work is supported by the STFC(UK), the Swedish Research Council, The German BMBF under grants no. 05P15RDFN1 and 05P12RDFN8, MINECO, Spain, under the grant FPA2014-57196-C5, Generalitat Valenciana, Spain, under the grant PROMETEOII/2014/019 and by the FEDER funds of the European Commission. This work has been supported by the European Community FP7 Capacities, contract ENSAR No. 262010."

\section{References}

[1] J. Beringer et al. (Particle Data Group), Phys. Rev. D 86, 010001 (2012).

[2] M. Thoenessen, B.M. Sherill, Nature 473, 25 (2011).

[3] R. Grzywacz et al., Phys. Lett. B 335, 439 (1995).

[4] M. Pfützner et al., Phys. Lett. B 444, 32 (1998).

[5] Zs. Podolyák et al., Phys. Lett. B 491, 225 (2000).

[6] D. Bachelier et al., Phys. Lett. B 172, 23 (1986).

[7] A. Kelić et al., Phys. Rev. C 70, 064608 (2004).

[8] A.I. Morales et al., Phys. Rev. C 84, 011601(R) (2011).

[9] J. Benlliure et al., JPS Conf. Proc. 6, 020039 (2015).

[10] I. Vidaña et al., EPJ Web of Conferences 107, 10003 (2016).

[11] Zs. Podolyák et al., Phys. Rev. Lett. 117, 222302 (2016). 
[12] H. Geissel et al., Nucl. Inst. Meth. B 70, 286 (1992).

[13] S. Akkoyun et al., Nucl. Instrum. Meth. 668, 26 (2012).

[14] N. Lalović et al., Nucl. Inst. Meth. A 806, 258 (2016).

[15] Huo Junde, Nucl. Data Sheets 110, 2689 (2009).

[16] D. Rudolph et al., Phys. Rev. C 78, 021301(R) (2008).

[17] D. Yan, J. Huo, Nucl. Data Sheets 121, 1 (2014).

[18] E. Dafni et al., Phys. Lett. B 781 (1978).

[19] O. Tarasov, Nucl. Phys. A 734, 536 (2004).

[20] A.S. Goldhaber, Phys. Lett. B 53, 306 (1974).

[21] E.C. Simpson et al., Phys. Rev. C 79064621 (2009).

[22] M. Pfützner et al., Phys. Rev. C 65, 064604 (2002).

[23] M. Bowry et al., Phys. Rev. C 88, 024611 (2013).

[24] E.C. Simpson et al., Phys. Rev. C 80, 064608 (2009).

[25] E.C. Simpson et al., Phys. Rev. C 82, 037602 (2010).

[26] Zs. Podolyák et al., Phys. Lett. B 672, 116 (2009).

[27] A.M. Denis-Bacelar et al., Phys. Lett. B 723, 302 (2013). 